\title{
Una estimación del producto potencial de Nicaragua, mediante el filtro de Hodrick - Prescott
}

\section{An estimate of the potential product of Nicaragua, using the Hodrick - Prescott filter}

Oslund Rains Franklin Sam ${ }^{1}$

\section{Resumen}

Se ha realizado una estimación de la producción potencial y la brecha del producto de Nicaragua utilizando el filtro de Hodrick-Prescott (1980), con datos de los informes del Banco Central de Nicaragua, tomando como referencia los años 1980-2014. Los resultados de la estimación demuestran que el nivel de la producción está por encima del producto potencial lo que presiona los precios al alza.

Palabras clave: Producto potencial; Producto Interno Bruto; Inflación; filtro de Hodrick-Prescott.

\section{Abstract}

An estimate of the potential production and output gap of Nicaragua has been made using the HodrickPrescott filter (1980), with data from the reports of the Central Bank of Nicaragua, taking the years 19802014 as a reference. The estimation results show that the level of production is above potential product, which puts upward pressure on prices.

Keywords: Potential output; Gross Domestic Product (GDP); Inflation; Hodrick - Prescott filter.

\section{Introducción}

El objetivo de este trabajo es presentar una estimación del producto potencial y la brecha del producto en Nicaragua en el período 1980-2014, utilizando los filtros de Hodrick-Prescott (1980), en cuanto que el producto potencial es una variable no observable, significa que se debe estimar y proyectar para calcular posteriormente la brecha del producto, que resulta de la diferencia entre el producto potencial y el producto real. Esto permite a los hacedores de políticas económicas tener una guía sobre la capacidad máxima de producción y fijar objetivos de crecimiento económico y estabilización de la economía.

Sin embargo, a veces la producción efectiva del país puede ser temporalmente mayor que la potencial. Estas tasas de utilización, mayores de lo normal, no pueden mantenerse indefinidamente, debido en parte a que los trabajadores no pueden hacer horas extraordinarias todas las semanas y las maquinarias a veces debe detenerse para ser mantenida y reparada. La producción potencial no es una cantidad fija, sino que crece con el paso del tiempo, debido al aumento tanto de la cantidad de capital y de trabajo de que se dispone, como de su productividad.

Por consiguiente, se deben Identificar las fases del ciclo en que se encuentra la actividad productiva y, por lo tanto, deducir si el impulso de una política será pro cíclico o contra cíclico y sus factores tanto de crecimiento productivo, tecnológico, las capacidades de expansión en el mediano y largo plazo, prever las presiones inflacionarias y sus variables de solución en un avance de consolidación fiscal.

\footnotetext{
Doctor en Ciencias Económicas, Director de contrataciones y adquisiciones de la Universidad de las Regiones Autónomas de la Costa Caribe Nicaragüense (URACCAN), email: orains@hotmail.com; ID Orcid: https://orcid.org/0000-0002-7169-1967
} 


\section{Revisión de literatura}

El concepto de producto potencial sirve para analizar la medición de las expansiones y de las contracciones de la economía. La producción potencial que también es llamada Producto Interno Bruto (PIB), potencial o producción de pleno empleo y puede definirse como la cantidad máxima de producto que podría generar una economía usando al máximo su capacidad productiva y sin generar presiones inflacionarias (Okun, 1962).

\section{Filtro de Hodrick y Prescott}

Esta técnica desarrollada por Hodrick y Prescott (1980), conocida también como HP, se basa en la posibilidad de descomponer la serie en sus componentes cíclico y permanente, estimando la componente cíclica como la diferencia entre la serie observada y su componente permanente, y propone la suma de los cuadrados de las segundas diferencias como una medida de variabilidad de la componente permanente.

Además, por su simplicidad, es una de las grandes virtudes para una aplicación generalizada en series de tiempo. La hipótesis que se mantiene para el cálculo del producto potencial es que la componente del logaritmo natural de la serie debe variar suavemente, de manera que las tasas de crecimiento varíen poco, $\mathrm{y}$, además, que la componente cíclica no sea más que desviaciones entorno a la componente permanente. De esta forma filtra el logaritmo nacional de la serie extrayendo la componente (usando una transformación lineal) y obteniendo la componente cíclica simplemente como la diferencia entre la serie y su componente permanente.

El procedimiento minimiza la variabilidad de la tasa de crecimiento de dicho componente.

Haciendo $x_{t}$ la serie de Producto Interno Bruto (PIB), es posible expresarlo como la suma de dos partes, ciclo $\left(C_{t}\right)$ y tendencia $\left(P_{t}\right)$.

$$
\begin{aligned}
& \text { Entonces:: } x_{t}=C_{t}+P_{t} \text {, donde } t=1, \ldots T \\
& \qquad \min _{(p t) t=0, \ldots T+1} \sum_{t=1}^{T}\left(C_{t}-P_{t}\right)^{2}+\lambda \sum_{t=1}^{T}\left(p_{t+1}-p_{t}\right)-\left(p_{t}-p_{t-1}\right)^{2}
\end{aligned}
$$

Donde $\lambda$, es el parámetro de suavización, que sanciona la suma de las segundas diferencias del componente permanente. Mientras más grande sea lambda, obtendremos una tendencia más suave y cuando lambda sea lo suficientemente pequeño tendremos una tendencia más volátil.

Un valor pequeño de $\lambda$ produce una serie cercana a la original (si $\lambda=0$, ambas son idénticas) y uno elevado reduce la sensibilidad de la tendencia a las fluctuaciones aleatorias ( $\mathrm{si} \lambda=\alpha$ la tendencia se confunde con la tasa de crecimiento promedio de la serie) imponiéndose un comportamiento determinístico.

El valor de $\lambda$ define la varianza de la estimación del componente tendencial y esta cae a medida que aumenta el factor de ponderación.

Hodrick y Prescott (HP) recomiendan para series trimestrales valores equivalentes a $\lambda=1600$ y $\lambda=100$ para series anuales (Mendoza y Rendon, 1998).

En las literaturas de los ciclos económicos aparece un uso generalizado del valor $\lambda=1600$ para series trimestrales, para datos anuales nos encontramos con $\lambda=100$ (Englund-Persson-Svensson, 1992), $\lambda=100$ (Backus-Khoe, 1992) o $\lambda=10$ (Baxter-King, 1995), Domenech et al. (1997). En un boletín Europeo del Banco Central Europeo (1999) aparecen valores por defecto $\lambda=1600$ para datos trimestrales y $\lambda=14400$ para los mensuales.

El programa econométrico E-views recomienda por defecto emplear $\lambda=14000$. 


\section{Material y Métodos}

Se aplicó el filtro Hodrick-Prescott (HP) a la serie del Producto Interno Bruto (PIB) desde el año de 1980 a 2014 con frecuencia anual, publicado por el Banco Central de Nicaragua (BCN).

Para el cálculo de $\lambda$ lo igualamos a 100 para datos anuales, siguiendo las especificaciones y recomendaciones de Hodrick - Prescott (HP).

El programa econométrico utilizado para el análisis estadístico y la estimación del filtro de Hodrick Prescott (HP) se llama Gretl que es una aplicación diseñada para hacer estimaciones.

\section{Resultados y discusión}

Debido a que el producto potencial es una variable no observable, nunca existirá certeza sobre el valor exacto del mismo. Los cálculos siempre serán estimaciones y aproximaciones a la realidad.

A continuación, se presenta mediante gráfica los resultados obtenidos utilizando el filtro de Hodrick y Prescott (HP):

\section{Gráfica 1}
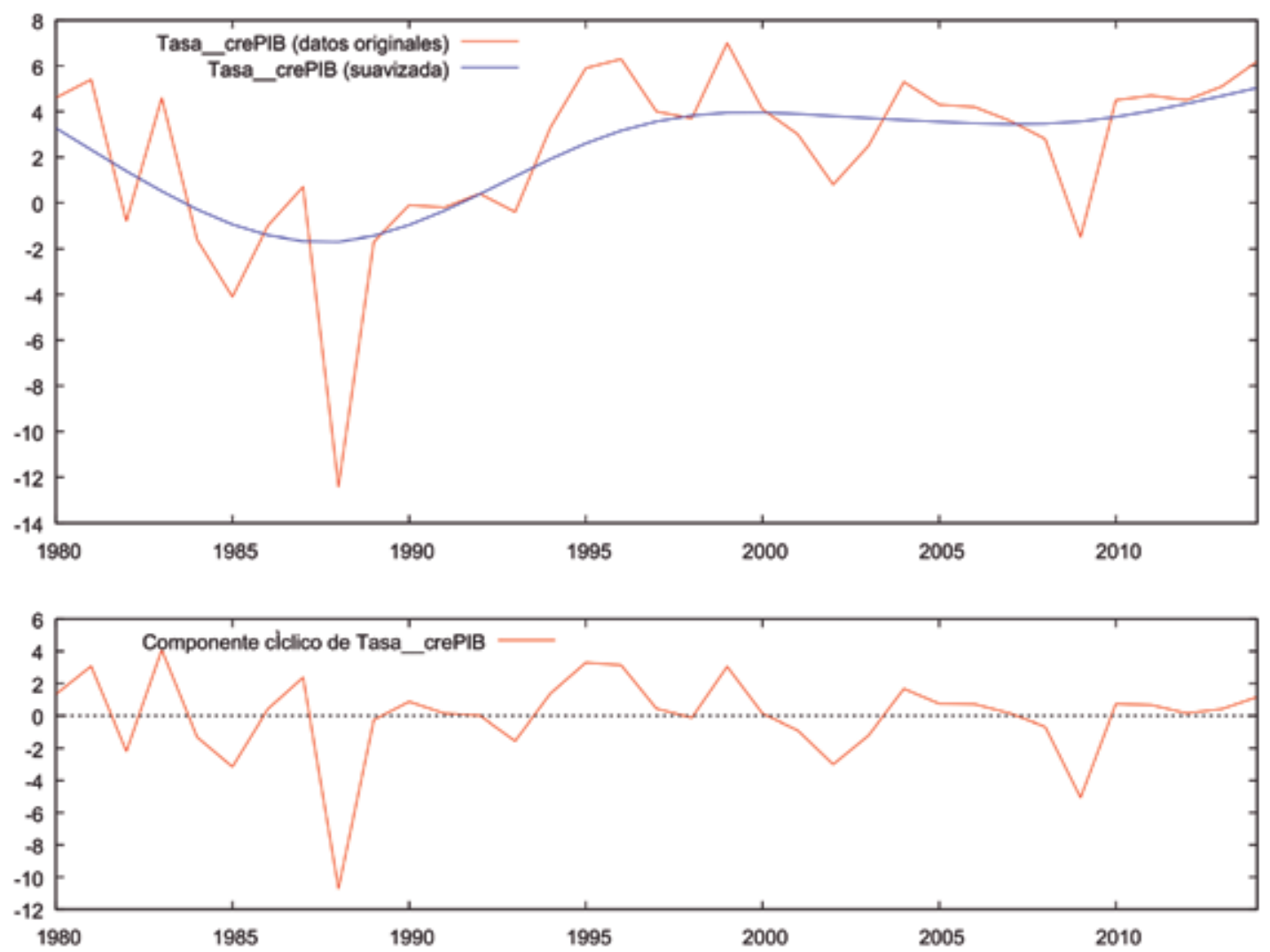

Fuente: Elaboración propia en base a datos del Banco Central de Nicaragua utilizando el programa Gretl.

Como se observa la evolución cíclica surge por el hecho de que el producto observado a menudo cae por debajo del producto potencial y esto posiblemente sea porque las empresas, instituciones y consumidores con sus producciones e ingresos son demasiadas bajas para proporcionar una salida a la producción potencial. A lo inverso sería que estas instituciones, empresas o consumidores intenten adquirir más bienes 
y servicios de lo que es capaz de producir la economía, empujando la producción efectiva por encima de su potencial. Cuando sucede esto, este proceso inestable puede tomar la forma de inflación pues la demanda excede a la oferta potencial.

Cuando el producto observable está por debajo del producto potencial, existe un problema de mercado insuficiente, la fuerza de trabajo y la capacidad instalada no está plenamente utilizada porque la demanda de sus productos no es suficientemente alta. Caso contrario cuando el producto potencial está por encima del producto observable se registran presiones inflacionarias.

La brecha del producto (diferencia entre el Producto Interno Bruto (PIB) observado y el Producto Interno Bruto (PIB) potencial es un indicador utilizado para predecir las presiones inflacionarias. Una brecha de producto positivo, es decir un nivel de producción por encima del producto potencial, tiende a presionar los precios al alza. Y es precisamente, lo que se ha presentado, (ver cuadro 1).

Por el conflicto militar que vivió el país (1974-1979 y 1981-1990), se dio una caída abrupta de la inversión privada y pública, y el país comienza a experimentar un alza generalizada de los precios, para posteriormente desembocar en una hiperinflación más prolongada de la historia. Desde 1985 la inflación promedio era de un poco más del 200\% para pasar en 1988 a un poco más del 33,000\% (BCN 2014).

Las presiones inflacionarias son un desequilibrio de oferta y demanda que provoca el ascenso en los precios en uno o varios mercados, y pueden ser de varios tipos: monetarias, fiscales, de costos, etc. Los mecanismos de propagación hacen que esos aumentos de precios se trasladen a otros mercados y se repitan a través del tiempo, posibilitando que la inflación se mantenga, aún luego de que hayan desaparecido los desequilibrios que la originaron. Ejemplos de mecanismos de propagación son las expectativas inflacionarias, o mecanismos de remarcación de precios basados en la inflación del período anterior.

No se debe descartar también las estructuras del mercado laboral que es ampliamente segmentado y heterogéneo. Gran parte de la población activa se encuentra sub empleada y la otra parte importante de ella se encuentra desempleada.

Cuando el Producto Interno Bruto (PIB) esté por encima de su nivel potencial, la inflación estará por encima del nivel de inflación anticipado, es decir, de la inflación esperada. La inflación, por lo tanto, se acelera. Caso contrario, cuando el PIB este por debajo de su nivel potencial, la inflación estará por debajo de la inflación anticipada, lo cual podría convertirse en una deflación.

Cuando el nivel de precios efectivo es mayor que el previamente esperado por los agentes, la inflación será mayor que la inflación esperada. Esto generará una producción por encima de su nivel potencial y consecuentemente, habrá una tasa de desempleo menor que la tasa natural. Y cuando la inflación efectiva es menor que la inflación esperada, la producción se encontrará por debajo de su nivel potencial, los costos de producción aumentarán y, por lo tanto, las empresas despedirán trabajadores, los cuales generara un desempleo por encima del nivel natural.

El Producto Interno Bruto (PIB) observado suele diferir del potencial y a la diferencia entre ambos se le denomina brecha de salida (output gap). Cuando el Producto Interno Bruto (PIB) está por encima de su potencial, se genera una brecha positiva, asociado con presiones inflacionista. Caso contrario cuando el Producto Interno Bruto (PIB) cae por debajo de su nivel potencial el output gap es negativo. Cuando esta brecha negativa es grande significa que la economía se encuentra en una crisis económica y que se ubica por debajo de su frontera de posibilidades de producción.

La frontera de posibilidades de la producción muestra varias combinaciones de producción que se puede alcanzar cuando todos los recursos se utilizan de la manera más eficiente, dado el estado actual de la tecnología. Por otro lado, la no significancia de la brecha de producto se puede deber al alto grado de desempleo existente en Nicaragua, que impide que aceleraciones en la demanda agregada genere presiones 
inflacionarias. Todo lo anterior implica que los factores que están detrás de la inflación de largo plazo en Nicaragua son la paridad de poder de compra y las presiones de costos (margen o markup).

En el cuadro 1, se muestra la estimación del Producto Interno Bruto (PIB) potencial y el residuo que en este caso es el GAP, todo en porcentajes.

\section{Cuadro 1}

Rango de estimación del modelo: 1980 - 2014

Desviación típica de los residuos $=3.7971$

\begin{tabular}{|c|c|c|c|c|}
\hline & Tasa_crePIB & Estimada & Residuo & \\
\hline 1980 & 4.60000 & 1.75629 & 2.84371 & \\
\hline 1981 & 5.40000 & 1.88683 & 3.51317 & \\
\hline 1982 & -0.800000 & 1.93034 & -2.73034 & \\
\hline 1983 & 4.60000 & 1.55324 & 3.04676 & \\
\hline 1984 & -1.60000 & 1.36469 & -2.96469 & \\
\hline 1985 & -4.10000 & 1.49522 & -5.59522 & \\
\hline 1986 & -1.00000 & 1.71278 & -2.71278 & \\
\hline 1987 & 0.700000 & 1.87232 & -1.17232 & \\
\hline 1988 & -12.4000 & 1.90133 & -14.3013 & * \\
\hline 1989 & -1.70000 & 2.24942 & -3.94942 & \\
\hline 1990 & -0.100000 & 2.13339 & -2.23339 & \\
\hline 1991 & -0.200000 & 2.69904 & -2.89904 & \\
\hline 1992 & 0.400000 & 3.11965 & -2.71965 & \\
\hline 1993 & -0.400000 & 3.61279 & -4.01279 & \\
\hline 1994 & 3.30000 & 3.51126 & -0.211259 & \\
\hline 1995 & 5.90000 & 3.48225 & 2.41775 & \\
\hline 1996 & 6.30000 & 3.35172 & 2.94828 & \\
\hline 1997 & 4.00000 & 3.10515 & 0.894849 & \\
\hline 1998 & 3.70000 & 2.94561 & 0.754392 & \\
\hline 1999 & 7.00000 & 2.58301 & 4.41699 & \\
\hline 2000 & 4.10000 & 1.82881 & 2.27119 & \\
\hline 2001 & 3.00000 & 2.11889 & 0.881113 & \\
\hline 2002 & 0.800000 & 2.07538 & -1.27538 & \\
\hline 2003 & 2.50000 & 2.04637 & 0.453632 & \\
\hline 2004 & 5.30000 & 1.97385 & 3.32615 & \\
\hline 2005 & 4.30000 & 1.84331 & 2.45669 & \\
\hline 2006 & 4.20000 & 1.78530 & 2.41470 & \\
\hline 2007 & 3.60000 & 1.88683 & 1.71317 & \\
\hline 2008 & 2.80000 & 1.91583 & 0.884167 & \\
\hline 2009 & -1.50000 & 2.22041 & -3.72041 & \\
\hline
\end{tabular}




\begin{tabular}{|l|l|l|l|l|}
\hline & \multicolumn{1}{|c|}{ Tasa_crePIB } & \multicolumn{1}{c|}{ Estimada } & \multicolumn{1}{c|}{ Residuo } & \\
\hline 2010 & 4.50000 & 2.16240 & 2.33760 & \\
\hline 2011 & 4.70000 & 1.94484 & 2.75516 & \\
\hline 2012 & 4.50000 & 1.88683 & 2.61317 & \\
\hline 2013 & 5.10000 & 1.88683 & 3.21317 & \\
\hline 2014 & 6.20000 & 1.85782 & 4.34218 & \\
\hline
\end{tabular}

Nota: * denota un residuo superior a 2.5 desviaciones típicas

Fuente: Datos de Informes del Banco Central de Nicaragua (BCN) y cálculo en base al programa Gretl.

La brecha presenta movimientos importantes alrededor del conflicto militar que vivió el país (19741979 y 1981-1990), lo que nos indica que la economía presenta una memoria larga y los efectos persisten en el tiempo. Además, como se mencionó anteriormente, cuando el nivel de producción está por encima del producto potencial tiende a presionar los precios al alza, o lo que es lo mismo cuando la estimación de la brecha del producto es positiva.

\section{Conclusiones}

Conocer el Producto Interno Bruto (PIB) potencial y la brecha del producto permite, entre otras cosas:

Identificar las fases del ciclo en que se encuentra la actividad productiva y, por lo tanto, deducir si el impulso de una política será pro cíclico o contra cíclico.

1. Determinar la contribución de los factores productivos y del avance tecnológico al crecimiento secular de la economía.

2. Estimar la capacidad de expansión del producto en el mediano plazo.

3. Anticipar la evolución de las presiones inflacionarias y proveer posibles situaciones de sobrecalentamiento.

4. Comprobar objetivamente los avances que se vayan logrando en materia de consolidación fiscal.

5. Caracterizar los ciclos económicos y fechar, asimismo, las fases recesivas y expansivas de la actividad productiva.

\section{Recomendaciones}

Para el producto potencial se recomienda considerar especificidades de tipo estructural, como las características del mercado que es ampliamente segmentado y heterogéneo. Gran parte de la población activa se encuentra sub empleada y, otra parte de ella desempleada. Estimular la inversión tanto pública como privada a través de deducciones tributarias y de tasas de interés subvencionadas directamente por el Estado.

\section{Agradecimiento}

Esta publicación obtuvo el financiamiento de: El Fondo de Asistencia Internacional de los Estudiantes y Académicos Noruegos (SAIH). 


\section{Lista de referencias}

Backus, D.K., Kehoe, P.J. (1992). International Evidence on the Historical Properties of Business Cycle (Evidencia internacional sobre las propiedades históricas del ciclo comercial). The American Economic Review, 82, pp. 864-888.

Baxter, M., King, R.G. (1995). Measuring Business Cycles: Approximate Band-pass Filters for Economic Time Series (Medición de ciclos comerciales: filtros aproximados de paso de banda para series de tiempo económicas). NBER. Working Paper 5022.

Boletín mensual del Banco Central Europeo (julio 1999).

BCN (2014). Nicaragua en Cifras. Informe del Banco Central de Nicaragua.

Bernanke B. S., Frank R. H (2007). Principios de Economía. Aravaca, Madrid: José Ignacio Fernández Soria.

Doménech, R., Gomez, V., Taguas, D. (1997). Filtering Methods Revised (Revisión de métodos de filtración). Documento de Trabajo de la Dirección General de Análisis y Programación Presupuestaria del Ministerio de Economía y Hacienda.

Englud, P., Persson, T., Svensson, L.E.O. (1992). Swedish Business Cycles 1861-1988 (Los ciclos económicos suecos 1861-1988). Journal of Monetary Economics, 30, pp. 343-371.

Franklin O. (2016). Aproximación a la relación PIB empleo desempleo (tesis doctoral). Universidad del Zulia, Venezuela.

Hodrick R. J, Prescott E. C (1980). Postwar U.S. Business Cycles: An Empirical Investigation. Carnegie Mellon University discussion paper no. 451 (Ciclos Comerciales de los Estados Unidos de la posguerra: una investigación empírica. Documento de discusión de la Universidad Carnegie Mellon no. 451).

Mendoza, M. Á., Rendón T., A. (1998). Ciclo de ventas empresariales y crecimiento económico en México. Revista Comercio Exterior, volumen 48, No 9. México.

Okun, A. (1962). Potential GDP: Its Measurement and Significance. Proceedings of the Business and Economic Static Section, American Statistical Association, 98-104. (PBI potencial: su medida y significado. Procedimientos de la Sección estática comercial y económica, American Statistical Association, 98-104). Reimpreso en Arthur Okun, The Political Economy of Prosperty, Brooking, Washington D.C, 1970, 132,145 . 